\title{
Editorial
}

\section{Atmospheric Boundary-Layer Processes and Atmospheric Modeling}

\author{
D. Bala Subrahamanyam, ${ }^{1}$ Ismail Gultepe, ${ }^{2}$ Sultan Al-Yahyai, ${ }^{3}$ and A. N. V. Satyanarayana ${ }^{4}$ \\ ${ }^{1}$ Space Physics Laboratory, Vikram Sarabhai Space Centre, Department of Space, Government of India, \\ Indian Space Research Organisation, Thiruvananthapuram 695022, India \\ ${ }^{2}$ Environment Canada, Meteorological Research Division, Cloud Physics and Severe Weather Research Section, \\ Toronto, ON, Canada M3H 5 T4 \\ ${ }^{3}$ Public Authority for Civil Aviation, Oman National Meteorological Services, Muscat, Oman \\ ${ }^{4}$ Centre for Oceans, Rivers, Atmosphere and Land Sciences, Indian Institute of Technology Kharagpur, Kharagpur 721302, India \\ Correspondence should be addressed to D. Bala Subrahamanyam; subrahamanyam@gmail.com
}

Received 31 December 2014; Accepted 31 December 2014

Copyright (C) 2015 D. Bala Subrahamanyam et al. This is an open access article distributed under the Creative Commons Attribution License, which permits unrestricted use, distribution, and reproduction in any medium, provided the original work is properly cited.

The atmospheric boundary layer (ABL), extending upward from the earth's surface, is substantially influenced by energy and moisture from the underlying surface through turbulent transport and physical processes [1]. More than $50 \%$ of the atmospheric kinetic energy is dissipated within this ABL [24]. Several boundary-layer processes are known to have a profound influence on the weather and climate system [5]. Appropriate representation of these processes in numerical atmospheric models and air quality models is accomplished partially or totally through different $\mathrm{ABL}$ parametrization schemes. Despite considerable progress in the field of atmospheric modelling in the past few decades, the errors and uncertainties associated with the ABL parameterization schemes remain one of the primary sources of inaccuracies in numerical weather prediction (NWP) model simulations. Thus, the underlying physics behind the boundary-layer processes and their accurate representation in numerical atmospheric models remain a subject of investigation for many researchers.

The skill and the performance of NWP models are of vital interest as far as the qualification of the model forecast products is concerned. Based on these, three thrust core areas are identified in the development of NWP models: (i) numerical aspects; (ii) physical aspects, including surface and ABL processes; and (iii) analysis and initialization procedures as well as measurements. Following a consistent progress in the field of weather and climate science, the Board of Atmospheric Sciences and Climate, National Research Council (NRC), USA, had envisaged a visionary statement in 1998, stating "Improvements in atmospheric observations, further understanding of atmospheric processes, and advances in technology will continue to enhance the accuracy and resolution of atmospheric analysis and prediction. As a consequence, society will enjoy greater confidence in atmospheric information and forecasts and will be able to act more decisively and effectively" [6]. Following the recent trends in the field of ABL processes and its linkage in the NWP models, we present a special issue on the atmospheric boundary-layer processes and atmospheric modeling. The main objectives of this special issue are to provide the scientific community with a compendium of some of the recent developments in the understanding of ABL processes and their representation in the NWP models. There was an overwhelming response from the researchers across the globe and finally all the accepted articles for publication cover a wide range of topics of growing importance in our understanding of $\mathrm{ABL}$ processes and their impacts on the NWP science. Though it was not possible to cover all the aspects of these fields, the accepted articles for publication indirectly reflect the recent advances in the area of NWP science and ABL processes.

The authors have extensively made use of the state-of-the art WRF (weather research and forecasting) model for exploring various aspects with regard to the NWP applications. S. Payra and M. Mohan used the WRF model for prediction 
of fog over the Indian region through a multirule based diagnostic approach, and they could discriminate the foggy and nonfoggy days with a reasonable accuracy. In a forecast application study, Yang et al. demonstrated the potential of WRF model in prediction of mesoscale convective systems through a case study in which they assimilated the Chinese Doppler radar and lightning data.

Using a WRF-based simulation approach, Y. Zheng et al. presented a series of numerical studies for investigation of the ABL structure, ambient wind, and pollutant source location and their impacts on the wind field and pollutant distribution within the built-up areas of Shenyang, China. L. Wen utilized the potential of the WRF model coupled with a Community Land Model for investigation of the impacts of the Great Salt Lake and its salinity on precipitation patterns. Y. Miao et al. used the WRF model for studying the effects of topography and urbanization on the local atmospheric circulations over Beijing-Tianjin-Hebei, China.

M. Moroni and A. Cenedese conducted intense laboratory simulations of local winds in the $\mathrm{ABL}$ through an image analysis technique by ignoring the synoptic winds and Coriolis effects and could satisfactorily explain the observed local winds. J. L. Palau and F. Rovira made use of 15-year long-term NCEP/NCAR reanalysis database for studying the mesoalpha scale tropospheric interactions within the Western Mediterranean Basin. Zhang et al. studied the spatial and temporal variability of aerosol vertical distribution based on LIDAR observations over Jinhua Basin of China. They also presented the variations in the $\mathrm{ABL}$ height obtained from their observations and compared them with concurrent measurements from the CALIPSO. G. Jocher et al. made use of eddy covariance method and a hydrodynamic model approach for derivation of sensible heat flux. A comparison of the two methods is carried out with regard to the season and mean wind directions. S. Sodoudi et al. investigated the urban heat island effects in megacity Tehran. Their study suggested some feasible mitigation strategies which can be adopted for reduction in air temperatures and consequently saving energy.

All the papers in this special issue presented a collection of latest research results in the field of atmospheric boundarylayer processes and atmospheric modeling. We are quite optimistic that these articles would be of great interest to the readers of this journal and will lead to new challenges.

\section{Acknowledgments}

As guest editors of this special issue, we would like to thank all the authors for their contributions to this volume. Special mention of thanks and appreciation is due to the international panel of reviewers for making their expertise available for evaluation of the manuscripts.

\section{Bala Subrahamanyam Ismail Gultepe Sultan Al-Yahyai A. N. V. Satyanarayana}

\section{References}

[1] I. Gultepe, R. Tardif, S. C. Michaelides et al., "Fog research: a review of past achievements and future perspectives," Pure and Applied Geophysics, vol. 164, no. 6-7, pp. 1121-1159, 2007.

[2] S. P. Arya, Introduction to Micrometeorology, Academic Press, San Diego, Calif, USA, 2nd edition, 2001.

[3] J. R. Garratt, Atmospheric Boundary Layer, Cambridge University Press, Cambridge, UK, 1992.

[4] R. B. Stull, An Introduction to Boundary Layer Meteorology, Kluwer Academic Publishers, Dordrecht, The Netherlands, 1988.

[5] T. R. Oke, Boundary Layer Climates, Halsted Press, New York, NY, USA, 2nd edition, 1987.

[6] BASC, The Atmospheric Sciences: Entering the Twenty-First Century, Board of Atmospheric Sciences and Climate, National Research Council, Washington, DC, USA, 1998. 

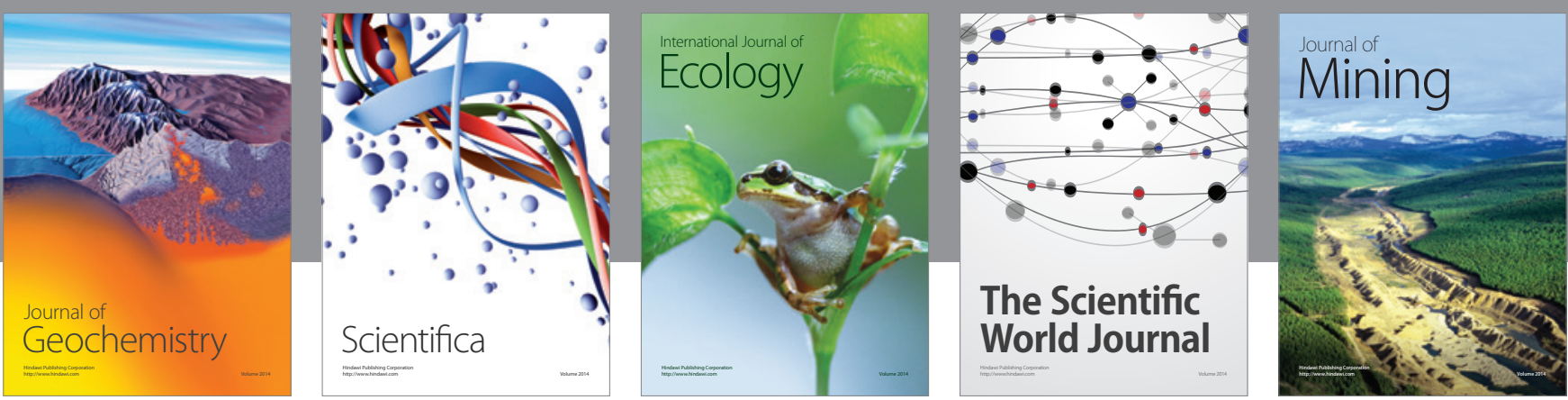

The Scientific World Journal
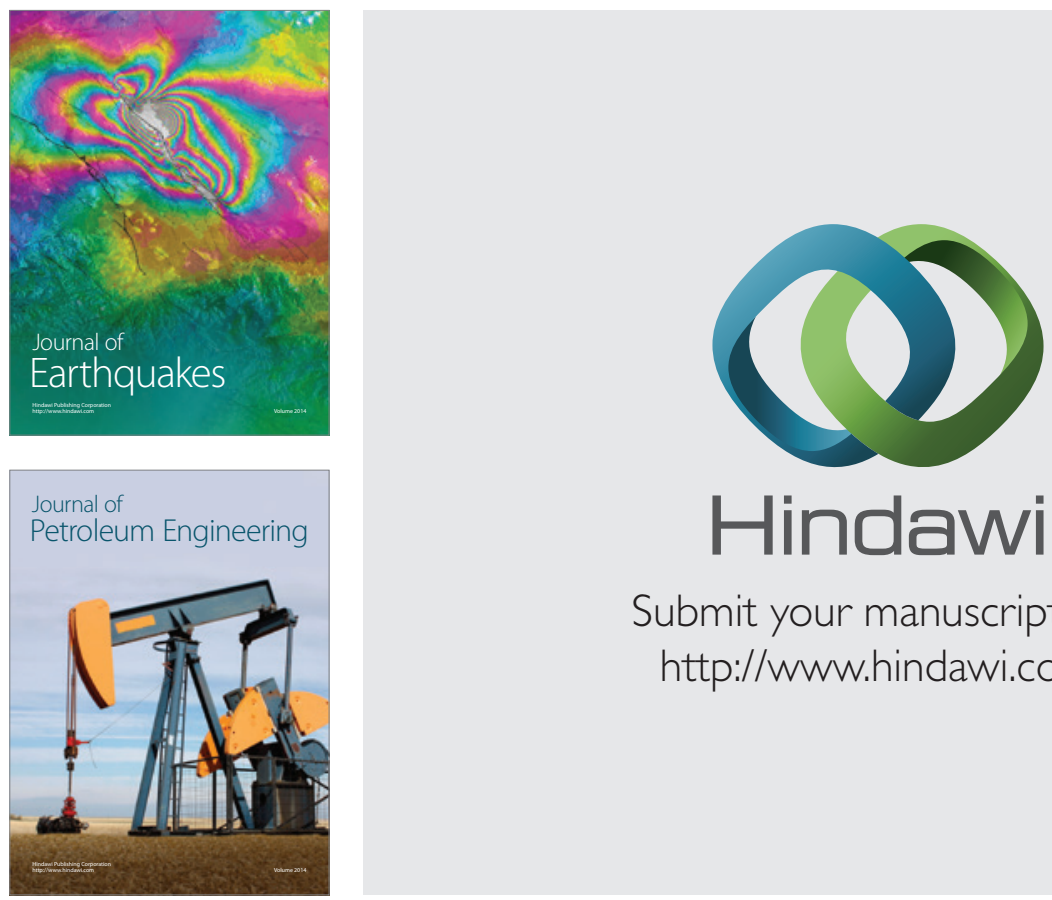

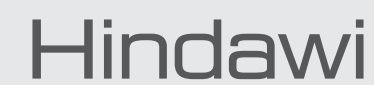

Submit your manuscripts at

http://www.hindawi.com
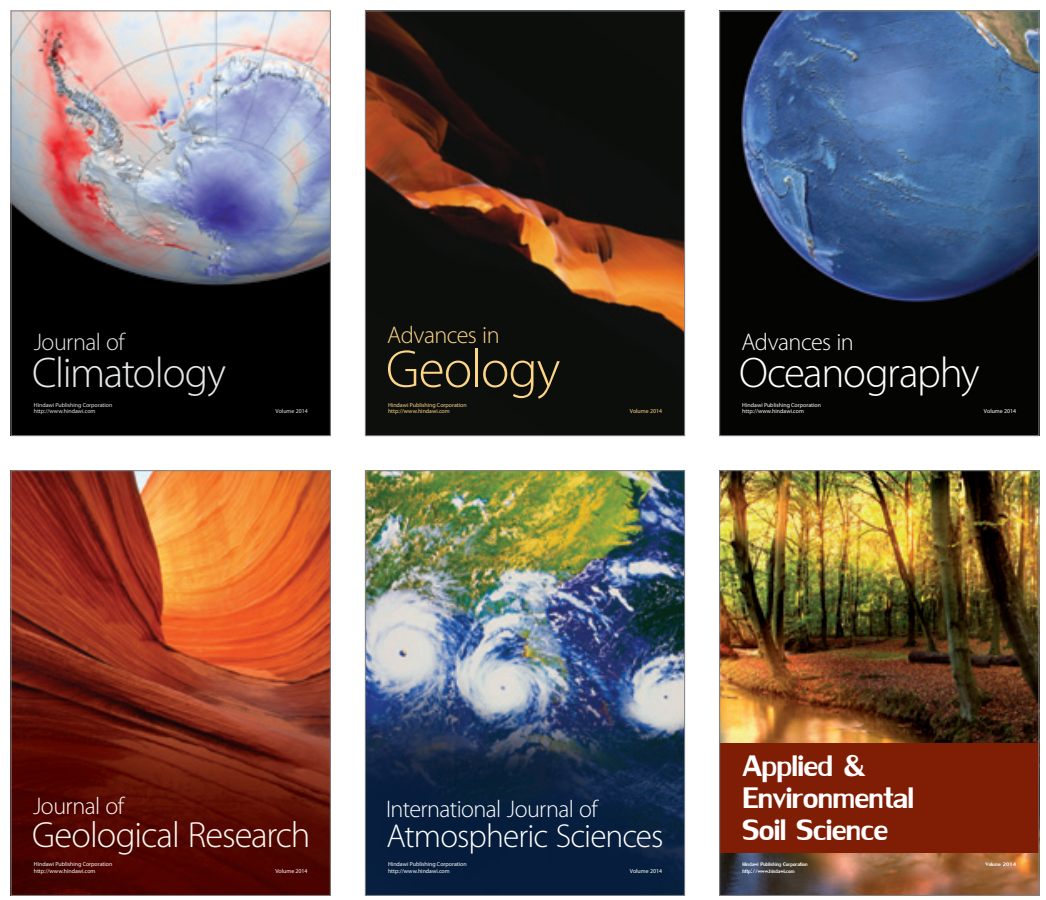
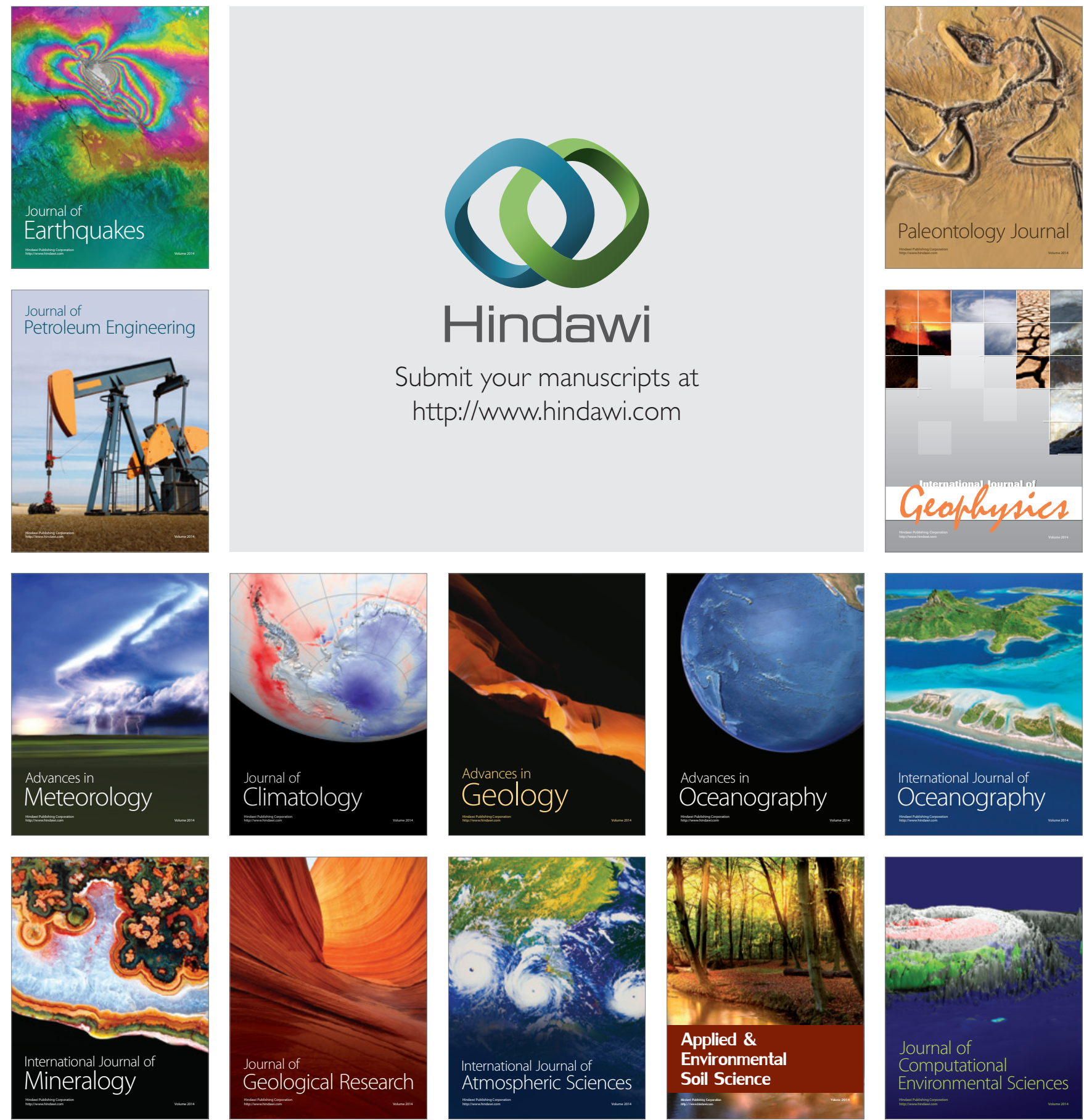